Manuelle Medizin 2010 • 48:470-470 DOI 10.1007/s00337-010-0801-z

Online publiziert: 28. November 2010

(c) Springer-Verlag 2010

\author{
R. Müller \\ Praxis für Allgemeinmedizin, Manuelle Medizin, Lehrpraxis der \\ Friedrich-Schiller-Universität Jena, Großengottern
}

\title{
Manuelle Medizin in der hausärztlichen Primärversorgung
}

Sehr geehrte Kolleginnen,

sehr geehrte Kollegen,

sehen Sie eine Zukunft für die manuelle Medizin?

Diese Frage können die meisten von uns spontan beantworten. Darüber hinaus wäre es von nicht unerheblichem Interesse zu wissen, wie Patienten, nicht manualtherapeutisch tätige Kollegen und Kostenträger diese Schicksalsfrage beantworten würden.

Nach Veröffentlichungen der Krankenkassen zeigt sich bei Patienten mit Erkrankungen des Bewegungssystems eine Zunahme der Krankheitshäufigkeit, insbesondere der Dauer der Arbeitsunfähigkeit. Zudem werden diese Patienten mit zunehmender Lebenserwartung multimorbider und die Funktionsstörungen komplexer. Andererseits werden sich die Ressourcen der Kostenträger in absehbarer Zeit nicht erweitern.

Dieser häufig diskutierten „Krise im System“ können wir Therapeuten nur mit effektiveren Behandlungsstrategien begegnen. Das bedeutet aus meiner Sicht, in kürzester Zeit die richtige Diagnose $\mathrm{zu}$ stellen und die erfolgversprechendste Therapie einzuleiten.

Beim Hausarzt sind die von den $\mathrm{Pa}$ tienten beklagten Beschwerden in der Regel sehr komplex. Aus eigener Erfahrung sind ca. zwei Drittel aller beklagten Schmerzen von myofaszialen Funktionsstörungen begleitet und könnten durch die Verfahren der manuellen Medizin effektiv behandelt werden. Das heißt, in der hausärztlichen Routine werden täglich
Patienten behandelt, deren Beschwerden eine Indikation zur manuellen Therapie darstellen.

Die Weichen zur Manualtherapie werden also in allererster Linie beim Hausarzt gestellt. Das würde konsequenterweise bedeuten, dass jeder Hausarzt mit den diagnostischen und therapeutischen Möglichkeiten der manuellen Medizin vertraut sein sollte. In Thüringen besitzen ca. 10\% der Hausärzte eine Zusatzqualifikation Manuelle Medizin. In Gesprächen mit interessierten nicht manualtherapeutisch tätigen Kolleginnen und Kollegen wird häufig eine unzureichende Kenntnis über die Diagnostik- und Behandlungskonzepte der manuellen Medizin geäußert. Benötigt werden plausible Vorstellungen über Untersuchungsmethoden, Behandlungsindikationen und Nebenwirkungen manualtherapeutischer Verfahren.

\section{- Es liegt somit an uns, unsere Arbeit nicht nur erfolgreich, sondern auch transparent zu gestalten.}

Das erfordert eine Diskussion darüber, welche relevanten klinischen Befunde in der Hausarztpraxis eine Indikation zur manuellen Medizin darstellen. Benötigt werden Behandlungskonzepte, die speziell auf den Zeitrahmen der Primärversorgung mit 60 bis 80 , teilweise bis 110 Patientenkontakten täglich zugeschnitten sind. Um die Qualität unserer Arbeit in die öffentliche Diskussion einzubringen, müssen wir trotz aller Kom- plexität objektivierbare Behandlungsziele definieren, die neben der Schmerzlinderung auch Parameter, wie die Verkürzung der Behandlung- bzw. Arbeitsunfähigkeitsdauer und vor allem die Verhinderung der Chronifizierung berücksichtigen. Dazu sind erste Bemühungen im Gange. Wenn es darüber hinaus gelingt, manualmedizinische Inhalte in die Weiterbildung zum Facharzt für Allgemeinmedizin einzubringen, wäre ein weiterer wichtiger Schritt getan.

Ich sehe die Herausforderung für die Zukunft der manuellen Medizin in der Transparenz unserer Konzepte und der festen Etablierung in der hausärztlichen Primärversorgung. Um diese zu erarbeiten, lade ich vor allem die hausärztlich tätigen Manualtherapeuten ein.

\section{Korrespondenzadresse \\ Dr. R. Müller \\ Praxis für Allgemeinmedizin, \\ Manuelle Medizin, \\ Lehrpraxis der Friedrich-Schiller-Universität Jena Bahnhofstr. 12, 99991 Großengottern \\ dr.ralf.mueller@online.de}

